\title{
EXPERIMENTAL STUDY OF STABILITY AND TRANSITION OF SUPERSONIC BOUNDARY LAYER ON SWEPT WING AT MACH NUMBER 2-4
}

\section{N. V. Semionov, A. D. Kosinov, Yu. G. Yermolaev, and R.I. Kazantsev}

Institute of Theoretical and Applied Mechanics 4/1 Institutskaya Str., Novosibirsk 630090, Russia

\begin{abstract}
The paper is devoted to an experimental study of the laminarturbulent transition and the instability disturbances evolution in a threedimensional (3D) supersonic boundary layer on a swept wing with sharp and blunted leading edges at $\mathrm{M}=2-4$. The detailed data of the natural disturbances development are obtained. Characteristic zones of disturbances evolution are determined. It is found that for Mach numbers 2 and 2.5 measurements can be performed in the region of the linear stage of disturbances evolution, and the experimental data can be compared with the results of the calculations on the linear stability theory. The destabilization effect of leading edge bluntness was obtained. The statistical analysis and the analysis of amplitude-frequency spectra confirmed the existence of the mechanism of a secondary instability and its features were revealed in the supersonic flow of a swept wing.
\end{abstract}

\section{INTRODUCTION}

The problem of the transition to turbulence in $3 \mathrm{D}$ boundary layers is very important and very complicated. In a 3D case, together with the well-known TollmienSchlichting (T-S) waves that lead to turbulent transition in the two-dimensional boundary layers, there exist instability on the leading edge of a swept wing, stationary vortexes with axes directed along the outer streamlines, and some traveling waves (not $\mathrm{T}-\mathrm{S}$ waves). The development of all instability disturbances and their relative role in transition strongly depends on the environmental conditions. Most theoretical and experimental results on the stability of a 3D boundary layer are obtained for a subsonic flow. Some recent studies in this field are discussed in reviews $[1-4]$ and other papers. However, very few theoretical and experimental 
investigations of the supersonic 3D boundary layer stability have been fulfilled up to date.

Malik et al. [5] studied a secondary instability on stationary crossflow disturbances in a swept cylinder boundary layer at Mach number $\mathrm{M}=3.5$. The secondary analysis yields three unstable modes with the peak growth rate at frequencies about $100 \mathrm{kHz}, 1.05 \mathrm{MHz}$, and $970 \mathrm{kHz}$. The most unstable travelling crossflow disturbance has a peak frequency of about $50 \mathrm{kHz}$; therefore, the unstable frequency for the secondary instability is an order of magnitude higher than that of the travelling crossflow disturbance. Mielke and Kleiser [6] studied the laminar-turbulent transition in a 3D supersonic boundary layer by means of direct numerical simulation (DNS) using the temporal model. The linear stability analysis shows the dominance of the crossflow instability. The secondary instability analysis reveals a broad band of secondary unstable modes travelling in the streamwise direction. Catafesta et al. [7] experimentally and theoretically studied the transition on a swept wing model at $\mathrm{M}=3.5$. In [7], using the envelope $e^{N}$ method for the linear stability calculation, the $N$-factor was obtained and results were compared with the observed transition locations. Travelling disturbances with $N=13$ provide a good correlation with the transition data over a range of unit Reynolds numbers and angles of attack. Disturbances with frequencies $40-60 \mathrm{kHz}$ have the largest $N$ factors, and it is assumed that the transition is more likely caused by them. An attempt to predict the transition with accounts for all major stages was made theoretically by Choudhari et al. [8]. Numerical studies of the secondary instability, the transition prediction, and control for swept wing supersonic boundary layers were made by $\mathrm{Li}$ and Choudhari in [9], where the experimental configuration of [10] was modeled. Experiments [10] on the passive flow control by a distributed roughness were made at Mach number $\mathrm{M}=2.4$ on 73 degree swept wing with the thicknessto-chord ratio of $4 \% . \mathrm{Li}$ and Choudhari examined the evolution of travelling disturbances and the destabilization of high-frequency secondary instabilities in the presence of finite amplitude stationary crossflow vortices of a specified spanwise wavelength and varying initial amplitudes. The excitation of several modes of the secondary instability with frequencies about $1 \mathrm{MHz}$ was detected. The 3D boundary layer stability to the stationary disturbances in a linear formulation was investigated in [11]. It was found that the boundary layer became unsteady to the stationary mode when the crossflow reached a sufficiently small value, which was less than $1 \%$ of the external flow.

The stability of supersonic boundary layer on a swept wing was studied experimentally only in ITAM [12-14]. Experiments [12-14] were made at Mach number $\mathrm{M}=2$ on a 40 degree swept wing with the thickness-to-chord ratio of $7.8 \%$. The evolution of natural fluctuations in the boundary layer on a swept wing was studied by Semionov et al. [13]. It was shown that the character of distribution of the mean and fluctuating characteristics of the boundary layer is similar to the case of subsonic velocities. It was obtained at $M=2$ that the 
disturbances growth in a 3D boundary layer occurs much faster than in the flat plate case. The results of an experimental study of evolution of controlled disturbances on a swept-wing model for Mach number $\mathrm{M}=2$ were presented in [13, 14]. The wave characteristics of traveling waves were obtained. The evolution of disturbances at frequencies of 10,20 , and $30 \mathrm{kHz}$ is similar to the development of traveling waves for subsonic velocities. The angle of inclination of the wave vector for energy-carrying disturbances is directed across the flow, and the group-velocity vector is aligned with the steady cross-flow disturbance.

The linear stage of the cross-flow instability in relation to stationary and unsteady disturbances was investigated theoretically by Gaponov and Smorodsky [15]. A direct quantitative comparison of the theory with the present authors' experiments [13] was presented. A good agreement of the theory with measurements performed in T-325 has been obtained only for spanwise scales of cross-flow vortices. However, computed growth rates differ significantly from measurements. The principal cause of such discrepancy of theoretical and experimental data was nonlinearity. New experiments $[16,17]$ were made on a symmetrical wing with a 45 degree sweep angle, a 3 percent thick circular-arc airfoil at $\mathrm{M}=2$ and low unit Reynolds numbers. In this case, the distance from the leading edge to the point of transition lenghtens in some times in comparison with the previous experiments. It has allowed investigating in detail the disturbances evolution in the supersonic boundary layer on swept wing, especially at the initial linear stage. The detailed data of the disturbances development up to the transition location were obtained for the first time. Characteristic zones of the disturbances evolution are determined. The position of the instability region of the secondary flow is experimentally defined. Some features of disturbances evolution, characteristic only for a supersonic boundary layer are revealed.

This work is a continuation of previous studies $[16,17]$. Some new results of the experimental study of the stability of the supersonic boundary layer on a swept wing with sharp and blunted leading edges at different Mach numbers are presented. Also, the secondary instability of the supersonic boundary layer on a swept wing is under consideration.

\section{EXPERIMENTAL SETUP}

The experiments were conducted at the Institute of Theoretical and Applied Mechanics of the Siberian Division of the Russian Academy of Sciences in the M-325 supersonic wind tunnel with test-section dimensions $0.2 \times 0.2 \times 0.6 \mathrm{~m}$ at Mach numbers $\mathrm{M}=2-4$ at low unit Reynolds numbers. Mach number was changed with the step 0.5 . The model length was $0.4 \mathrm{~m}$, its width was $0.2 \mathrm{~m}$, and the maximum thickness was $12 \mathrm{~mm}$. Edges of the model had a different bluntness. The radius of the edge bluntness was equal $r=1 \mathrm{~mm}$ and another leading edge 
was approximately $0.1 \mathrm{~mm}$. The model was mounted at zero incidences in the central section of the test section of the wind tunnel. Photo of the model in the test section was presented in [16].

The oscillations were measured by a constant-temperature hot-wire anemometer. Single-wire tungsten probes of $5 \mu \mathrm{m}$ (or $10 \mu \mathrm{m}$ ) in diameter and $1.2 \mathrm{~mm}$ in length were used. The overheat ratio of the wire was 0.8 , and the measured disturbances corresponded to mass-flow fluctuations. The fluctuating and mean characteristics of the flow were measured by an automated data acquisition system. The fluctuation signal from the hot-wire anemometer was measured by a 12-bit A/D converter with a digitization on time $1.33 \mu \mathrm{s}$, and a mean voltage was fixed by a voltmeter. The length of each realization was 65536 points. With the help of the discrete Fourier transform on time t the amplitude-frequency spectra were determined:

$$
e_{f}^{\prime}\left(x^{\prime}, z^{\prime}, y\right)=\frac{2}{T} \sum_{k} e^{\prime}\left(x^{\prime}, z^{\prime}, y, t_{k}\right) \exp \left[i \omega t_{k}\right] \Delta t_{k}
$$

where $T$ is the length of the digital time trace; $\Delta t_{k}=t_{k-1}-t_{k}$; and $e^{\prime}\left(x^{\prime}, z_{j}^{\prime}, y, t_{k}\right)$ is the digital oscillogram of a pulsation signal from a hot-wire anemometer. The values of the mass flow fluctuations $\rho U$ were determined by the method described in [18]. To measure a transition position, a hot-wire sensor was used.

To determine the nonlinear interaction of perturbations, a well-known fact was used that the Gaussian signal indicate a linear process (linear independence of the harmonic components of the signal), and any significant deviation from the normal distribution shows a nonlinearity of the process [19-22]. Evaluation for the normality of the probability density distribution was carried out according to [23]. The estimates of "skewness" and "kurtosis" of the measured pulsation signals were made:

- central moment of $k$-order:

$$
m_{k}=\frac{1}{N} \sum_{i=1}^{N}(x(i)-\bar{x})^{k}
$$

- parameter of skewness:

$$
g_{1}=\frac{m_{3}}{m_{2}^{3 / 2}}
$$

- parameter of kurtosis:

$$
g_{2}=\frac{m_{3}}{m_{2}^{2}}-3
$$




\section{RESULTS AND ANALYSIS}

The evolution of natural disturbances in the supersonic boundary layer of a swept wing was investigated in detail. Oscillograms, amplitude-frequency spectra, mean velocity profiles, pulsation profiles and statistical diagrams of natural fluctuations were obtained. Measurements were made at Mach number $\mathrm{M}=2$ and unit Reynolds number $\operatorname{Re}_{1}=5 \cdot 10^{6} \mathrm{~m}^{-1} ; \mathrm{M}=2.5$ and $\operatorname{Re}_{1}=5 \cdot 10^{6} \mathrm{~m}^{-1}$, $\operatorname{Re}_{1}=10 \cdot 10^{6} \mathrm{~m}^{-1}$, and $\operatorname{Re}_{1}=17.5 \cdot 10^{6} \mathrm{~m}^{-1} ; \mathrm{M}=3$ and $\operatorname{Re}_{1}=5 \cdot 10^{6} \mathrm{~m}^{-1}$ and $\operatorname{Re}_{1}=6.6 \cdot 10^{6} \mathrm{~m}^{-1} ; \mathrm{M}=3.5$ and $\operatorname{Re}_{1}=7.3 \cdot 10^{6} \mathrm{~m}^{-1}$; and $\mathrm{M}=4$ and $\operatorname{Re}_{1}=10.2 \cdot 10^{6} \mathrm{~m}^{-1}$.

At the initial stage of the study, the curves of the growth of natural disturbances at different Mach numbers in the case of a sharp leading edge or the blunt leading edge were obtained. Measurements were performed in the critical layer, where the pulsations have a maximum value. When the hot-wire sensor was moved along the longitudinal coordinate $x$, the mean voltage $E$ in the diagonal of the bridge was kept constant by moving a probe along the normal coordinate $y$. Dependences of the mass flux vs. Reynolds number $\operatorname{Re}=\operatorname{Re}_{1} x$ (where $x$ is the longitudinal coordinate) for the case of a sharp leading edge are shown in Fig. 1a. Analogues distributions obtained for the case of a blunted leading edge are presented in Fig. $1 b$.

Maxima in the distributions correspond to the position of the laminarturbulent transition. It is seen that for all Mach numbers, the transition on the blunted model starts earlier. The minimum value of the transition Reynolds number $\mathrm{Re}_{\mathrm{tr}}$ was obtained for $\mathrm{M}=3.5$, which agrees qualitatively with the experimental transition results on a flat plate at the same facility. Note that at each point of measurements, the amplitude-frequency spectra and statistical dis-

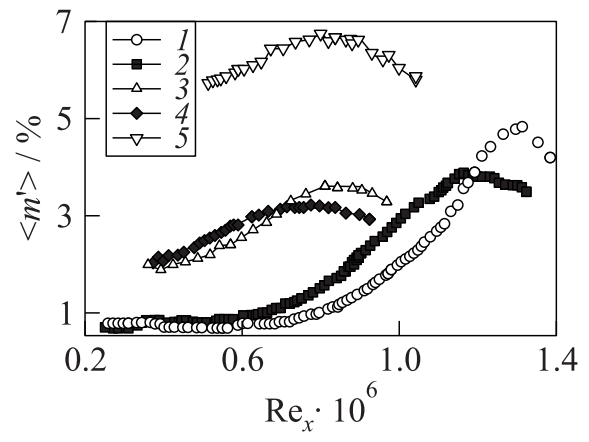

(a)

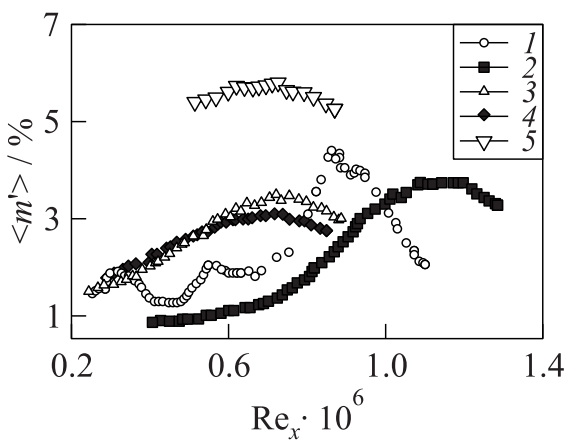

(b)

Figure 1 Dependences of mass flux pulsation $\left\langle m^{\prime}\right\rangle$ vs. Reynolds number Re for the cases of sharp $(a)$ and blunted $(b)$ leading edges: $1-\mathrm{M}_{\infty}=2.0, \operatorname{Re}_{1}=5 \cdot 10^{6} \mathrm{~m}^{-1}$; $2-\mathrm{M}_{\infty}=2.5, \mathrm{Re}_{1}=5 \cdot 10^{6} \mathrm{~m}^{-1} ; 3-\mathrm{M}_{\infty}=3.0, \mathrm{Re}_{1}=6.6 \cdot 10^{6} \mathrm{~m}^{-1} ; 4-\mathrm{M}_{\infty}=3.5$, $\operatorname{Re}_{1}=7.3 \cdot 10^{6} \mathrm{~m}^{-1} ;$ and $5-\mathrm{M}=4.0, \operatorname{Re}_{1}=10.2 \cdot 10^{6} \mathrm{~m}^{-1}$ 
tributions were obtained too. With the help of statistical analysis it was found that for Mach numbers 2 and 2.5 measurements can be performed in the region of the linear stage of the disturbances evolution, and the experimental data can be compared with the results of the calculations on the linear stability theory. The experimental data obtained at $\mathrm{M}=2$ are discribed in detail in $[16,17]$. The position of the the instability region of the secondary flow at $M=2$ was experimentally defined, and the growth of traveling disturbances are observed at $\operatorname{Re}_{x} \geq 0.35 \cdot 10^{6}$. In a supersonic swept wing boundary layer in the region $0.35 \cdot 10^{6}<\operatorname{Re}_{x}<0.7 \cdot 10^{6}$ at $\mathrm{M}=2$ and in the region $0.25 \cdot 10^{6}<\operatorname{Re}_{x}<0.6 \cdot 10^{6}$ at $\mathrm{M}=2.5$, linear disturbances evolution was observed. At Mach 3, 3.5, and 4, nonlinear processes were observed from the beginning of the region of measurements (50 mm from the leading edge of the swept wing). Apparently, in this case, the same transition scenario is realized as at subsonic flow velocities at a high level of turbulence [1-4]. It is well known that for the subsonic case, unsteady cross-flow disturbances are dominated at medium-to-high-turbulence conditions where they are excited on higher amplitude levels and hinder the development of the unstable steady modes.

Note that for the case of blunt model, at $\mathrm{M}=2$, modulations in the distributions of the mass-flux pulsation in the boundary layer and the free flow over a swept wing model were marked. As it can be seen in Fig. $1 b$, nothing like this at other Mach numbers was observed. The question of such an unusual behavior at $\mathrm{M}=2$ is still open.

Measurements across the boundary layer were made for all the abovementioned Mach numbers. Obtained distributions of mass flux pulsation $\left\langle m^{\prime}\right\rangle$ in dependence on normal coordinate $y$ at $\mathrm{M}=2.5$ are presented in Fig. 2. Dependences of $\left\langle m^{\prime}\right\rangle(y)$ have two maxima, the first corresponding to the critical layer, the second - to the subsonic layer. Two maxima in dependences were

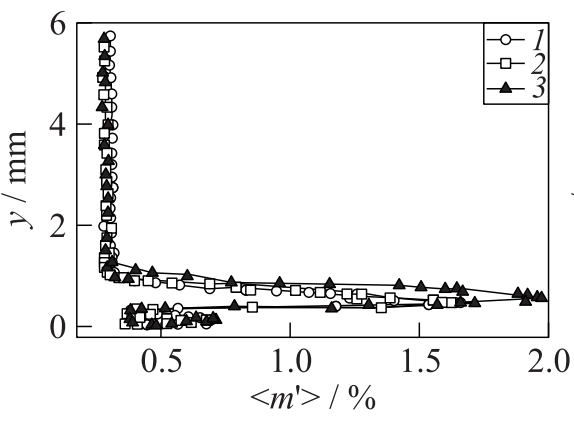

(a)

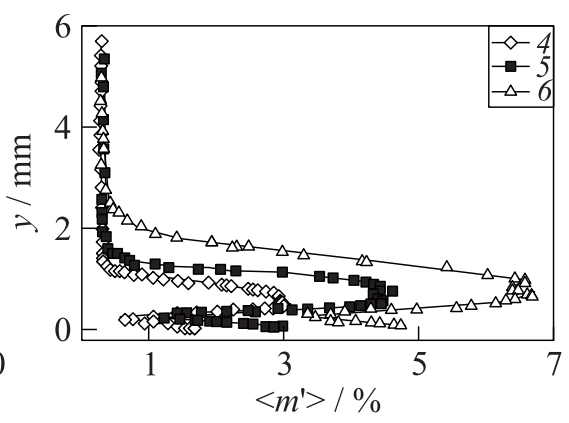

(b)

Figure 2 Distributions of mass flux pulsation $\left\langle m^{\prime}\right\rangle$ vs. normal coordinate $y$ at $\mathrm{M}=2.5$ and $\operatorname{Re}_{1}=5 \cdot 10^{6} \mathrm{~m}^{-1}: 1-x=75 \mathrm{~mm} ; 2-100 ; 3-125 ; 4-150 ; 4-175$; and $6-x=200 \mathrm{~mm}$ 
observed in the case of a flat plate, too. But in the supersonic boundary layer on the flat plate, the second maximum was less than the maximum in the critical layer. A fast growth of disturbances corresponding to the second maximum was observed in the case of the swept wing in the nonlinear region of the disturbances evolution.

It was found that the nature of the perturbations in the case of the blunt model mostly resembles the case of the sharp leading edge but at lower Reynolds numbers. An additional mode of perturbation was observed above the critical layer (see Fig. 2a). These perturbations are rapidly escalating, and, in the last sections, lead to a complex form of the main peak. Note that the presence of this mode of disturbances can be noticed in the profiles measured at Mach $2[16$, $17]$.

A statistical analysis was made for all experimental data obtained at $\mathrm{M}=2$ (for measurements in the critical layer of the streamwise disturbances evolution and for profile measurements). Figure 3 shows the examples of the results of the statistical analysis performed for the profiles of the pulsations.

Deviations of "skewness," "kurtosis" are close to zero and are the same as in the free stream for the profiles measured at $\operatorname{Re}_{x}=0.35 \cdot 10^{6}(x=70 \mathrm{~mm})$ and $\operatorname{Re}_{x}=0.6 \cdot 10^{6}(x=120 \mathrm{~mm})$, so we can say that processes are linear. The first significant deviation of the histograms obtained from a normal distribution was observed at $\operatorname{Re}_{x}=0.7 \cdot 10^{6}(x=140 \mathrm{~mm})$ above and below the critical layer. Note that when $\operatorname{Re}_{x}=0.7 \cdot 10^{6}$ in the vicinity of the second maximum, the processes are linear, but when $\operatorname{Re}_{x}=0.9 \cdot 10^{6}(x=180 \mathrm{~mm})$, the deviation from the normal distribution grows considerably. With increasing nonlinearity, disturbances begin to grow rapidly, especially in the vicinity of the second peak. And as noted earlier $[16,17]$, in the vicinity of the maximum near the surface of the model, the perturbations on the amplitude becomes comparable to the disturbances in the critical layer.

Amplitude-frequency spectra of disturbances in dependence on longitudinal coordinate $x$ at $\mathrm{M}=2$ and 2.5 are presented in Fig. 4 . The measurements were conducted in the layer of maximum fluctuations. At $\mathrm{M}=2$, with increasing of Reynolds number, there was an intensive excitation and growth of pulsations in the range of frequencies from 10 to $35 \mathrm{kHz}$ at the initial stage of disturbances and from 10 to $80 \mathrm{kHz}$ close to the transition location. The frequency ranges at $\mathrm{M}=2.5$ are wider, for example, from 10 to $45 \mathrm{kHz}$ at the initial stage of disturbances and from 10 to $200 \mathrm{kHz}$ close to the transition location. Further expanding of the range of frequencies was observed with increasing Mach number.

A comparison of the results of statistical analysis and analysis of amplitudefrequency spectra was performed. Fluctuations grow at the frequency range from 8 to $35 \mathrm{kHz}$ in the region of the linear evolution of disturbances at $\mathrm{M}=2$. Nonlinear processes lead to increasing of high-frequency disturbances. As is known [1, $2,9,24-26]$ for the subsonic boundary layer on a swept wing, at downstream vortex saturation, the mean flow is deformed which leads to the formation of 


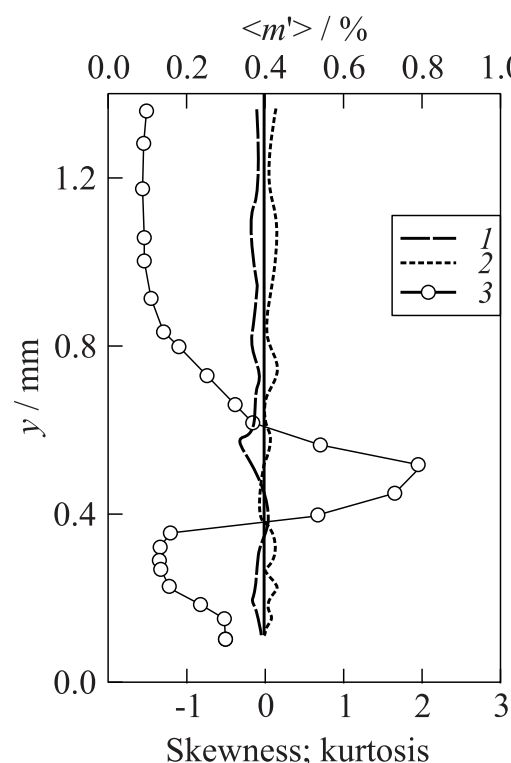

(a)

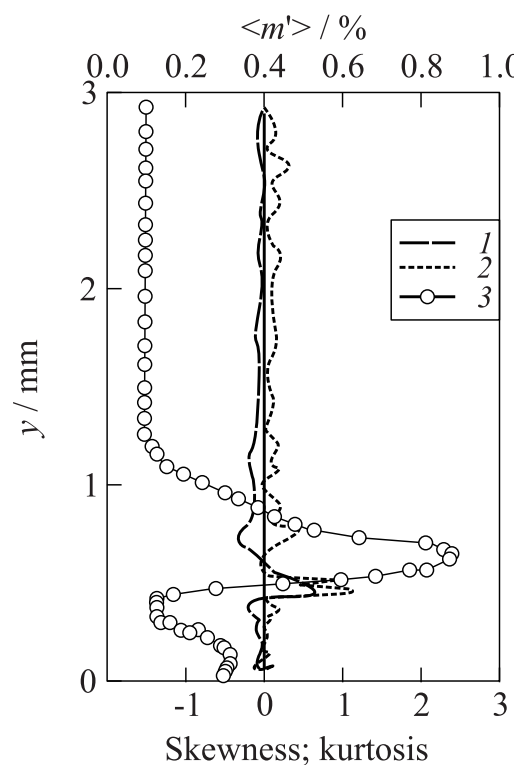

(c)

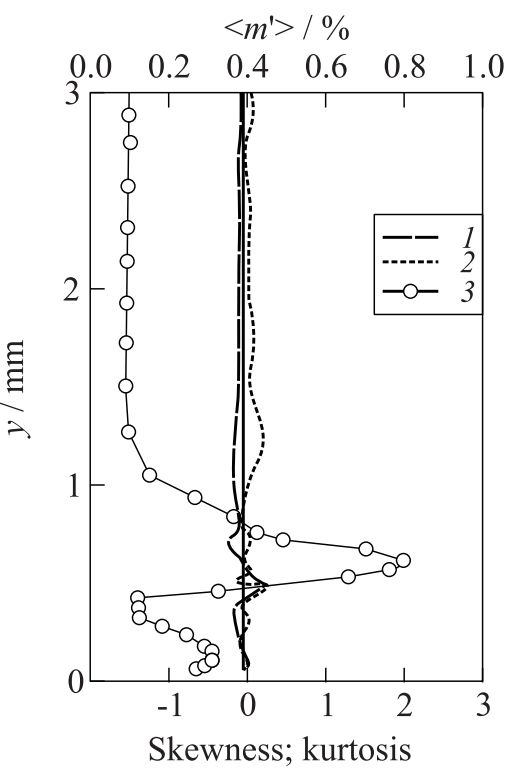

(b)

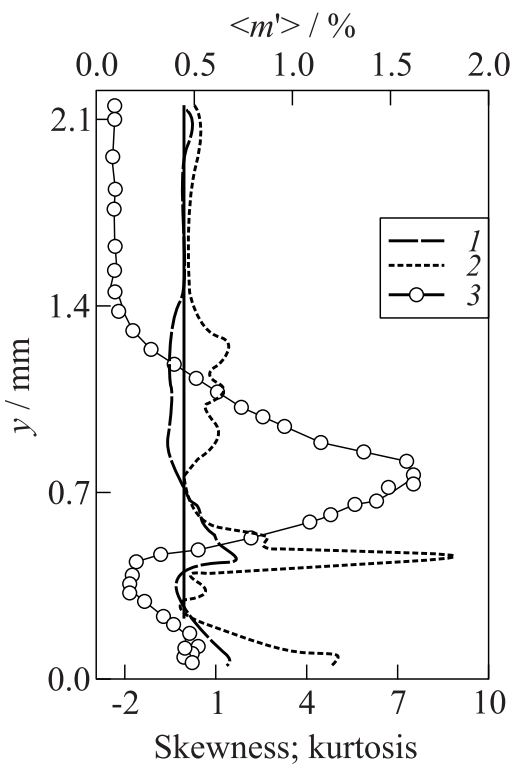

(d)

Figure 3 Dependences of "skewness" (1), "kurtosis" (2) and $\langle m\rangle(3)$ over normal coordinate $y$ for different $x$ positions at $\mathrm{M}=2$ and $\operatorname{Re}_{1}=5 \cdot 10^{6} \mathrm{~m}^{-1}:(a) x=70 \mathrm{~mm}$; (b) $120 ;(c) 140$; and $(d) x=180 \mathrm{~mm}$ 

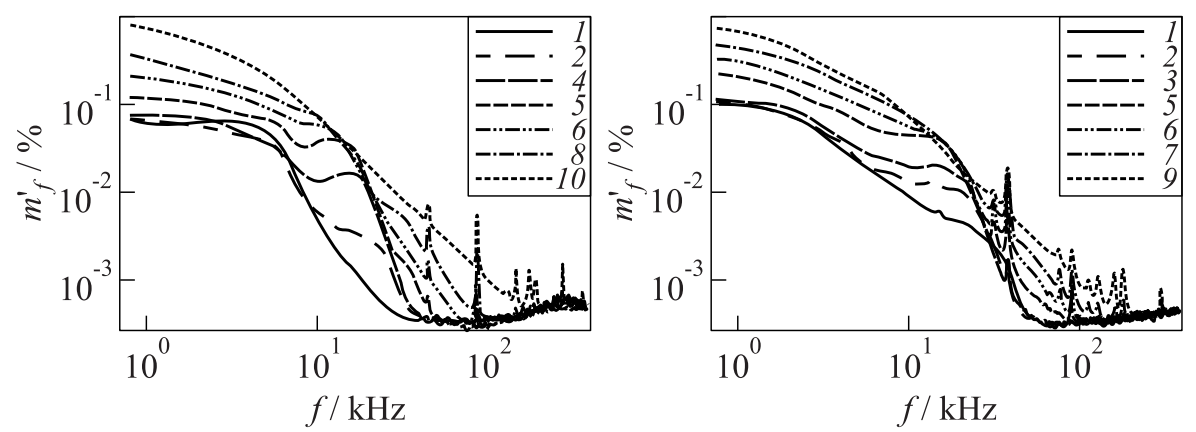

Figure 4 Amplitude-frequency spectra of disturbances at $\mathrm{M}_{\infty}=2.0(a)$ and $2.5(b)$ : $1-\operatorname{Re}_{x}=0.3 \cdot 10^{6} ; 2-0.5 \cdot 10^{6} ; 3-0.6 \cdot 10^{6} ; 4-0.7 \cdot 10^{6} ; 5-0.8 \cdot 10^{6} ; 6-$ $0.9 \cdot 10^{6} ; 7-1.0 \cdot 10^{6} ; 8-1.1 \cdot 10^{6} ; 9-1.2 \cdot 10^{6} ;$ and $10-\operatorname{Re}_{x}=1.3 \cdot 10^{6}$

strong shear flow. As a result, highly unstable conditions for inflectional instability start to appear both in $U(z)$ and $V(y)$ velocity profiles. Inflectional mean profiles trigger a convective high-frequency secondary instability with explosive spatial growth of unsteady modes. So, the secondary instability modes of crossflow vortices can be classified into $y$ - and $z$-modes. The frequency of the most amplified secondary mode is about one order of magnitude higher than the frequency of the primary cross-flow disturbances. The inflectional velosity profiles are realized in supersonic boundary layer on the swept wing, too. On the other hand, as obtained at subsonic speeds, the secondary instability is observed in the nonlinear stage of disturbances evolution. So, it can be assumed that the growth of high-frequency part of the spectrum $(f>35 \mathrm{kHz})$ is caused by the mechanism of secondary instability at supersonic speeds at $M=2$. Surely, this conclusion needs to be supported by theory. Similar results are obtained for other Mach numbers. Note that the excitation of several modes of secondary instability was detected theoretically in $[5,9]$. Modes with the peak growth rate at frequencies about $100 \mathrm{kHz}, 1.05 \mathrm{MHz}$, and $970 \mathrm{kHz}$ were detected in [5] at $\mathrm{M}=3.5$, and with frequencies about $1 \mathrm{MHz}$ were detected in [9] at $\mathrm{M}=2.4$. Unfortunately, a hot-wire anemometer cannot measure the perturbations with frequencies around $1 \mathrm{MHz}$. But the mode at $f$ about $100 \mathrm{kHz}$ in the swept cylinder is in good agreement with the authors' experimental data at $\mathrm{M}=3.5$.

\section{CONCLUDING REMARKS}

The evolution of natural disturbances in the supersonic boundary layer of a swept wing is investigated in detail for the cases of sharp and blunted leading edges. Oscillograms, amplitude-frequency spectra, pulsation profiles and statistical di- 
agrams of natural fluctuations are obtained. The profiles of pulsation have two maxima, the first corresponding to the critical layer and the second to the subsonic one. A fast growth of disturbances corresponding to the second maximum was observed in the case of swept wing in the nonlinear region of disturbances evolution. The position of the instability region of the secondary flow at $M=2$ and 2.5 is defined experimentally. A strong influence of the swept wing leading edge bluntness on the development of disturbances is observed.

The characteristic zones of the disturbances development are defined. It is found that for Mach numbers 2 and 2.5, the measurements can be performed in the region of the linear stage of the disturbances evolution, and the experimental data can be compared with the results of the calculations on the linear stability theory. At Mach 3, 3.5, and 4, nonlinear processes are observed from the very beginning of measurements ( $50 \mathrm{~mm}$ from the leading edge of the wing). With the help of the statistical analysis and the analysis of amplitude-frequency spectra the existence of the mechanism of the secondary instability is confirmed and its features are revealed in the supersonic flow of a swept wing. Fluctuations grow at the frequency range from 8 to $35 \mathrm{kHz}$ in the linear region. Nonlinear processes lead to increasing of high-frequency disturbances. It can be assumed that at $\mathrm{M}=2$, the growth of the high-frequency part of the spectrum $(f \geq 35 \mathrm{kHz})$ is caused by the secondary instability. Similar results are obtained for other Mach numbers.

\section{ACKNOWLEDGMENTS}

This work is supported financially by the RFBR under grants 08-01-00124, 0901-00767, and 11-01-00195.

\section{REFERENCES}

1. Arnal, D., G. Casalis, and J. C. Juillen. 1990. Experimental and theoretical analysis of natural transition on "infinite" swept wing. In: Laminar-turbulent transition. Springer-Verlag. 311-25.

2. Bippes, H. 1999. Basic experiments on transition in three-dimensional boundary layers dominated by crossflow instability. Prog. Aero. Sci. 35:363-412.

3. Boiko, A. V., G.R. Grek, A. V. Dovgal, and V.V. Kozlov. 2002. The origin of turbulence in near-wall flows. Berlin: Springer-Verlag.

4. Saric, W. S., H. L. Reed, and E. B. White. 2003. Stability and transition of threedimensional boundary layers. Ann. Rev. Fluid Mech. 35:413-40.

5. Malik, M. R., F. Li, and C.-L. Chang. 1996. Nonlinear crossflow disturbances and secondary instabilities in swept-wing boundary layers. In: Nonlinear instability and transition in three-dimensional boundary layers. Kluwer Acad. Publ. 257-66. 
6. Mielke, C., and L. Kleiser. 2000. Investigation of transition to turbulence in a 3D supersonic boundary layer. In: Laminar-turbulent transition. Springer-Verlag. 397-402.

7. Cattafesta, L. N. III , V. Iyer, J. A. Masad, R. A. King, and J. R. Dagenhart. 1995. Three-dimensional boundary-layer transition on a swept wing at Mach 3.5. AIAA J. 33:2032-37.

8. Choudhari, M., C.-L. Chang, C.L. Street, and P. Balakumar. 2003. Integrated transition prediction: A case study in supersonic laminar flow control. AIAA Paper No. 2003-0973.

9. Li, F., and M. Choudhari. 2011. Spatially developing secondary instabilities in compressible swept airfoil boundary layers. Theor. Comput. Fluid Dyn. 25(1-4):6584 .

10. Saric, W. S., and H. L. Reed. 2002. Supersonic laminar flow control on swept wings using distributed roughness. AIAA Paper No. 2002-147.

11. Asai, M., N. Saito, and N. Itoh. 2000. Instability of compressible three-dimensional boundary layer to stationary disturbances. Trans. Japan Soc. Aero. Space Sci. 43(142):190-95.

12. Ermolaev, Yu. G., A.D. Kosinov, V. Ya. Levchenko, and N. V. Semionov. 1995. Instability of a three-dimensional supersonic boundary layer. J. Appl. Mech. Techn. Phys. 36(5):50-54.

13. Semionov, N. V., Yu. G. Ermolaev, A. D. Kosinov, and V. Ya. Levchenko. 2003. Experimental investigation of development of disturbances in a supersonic boundary layer on a swept wing. Thermophys. Aeromech. 10(3):347-58.

14. Semionov, N. V., A. D. Kosinov, and Yu. G. Ermolaev. 2008. Evolution of disturbances in a laminarized supersonic boundary layer on a swept wing. J. Appl. Mech. Techn. Phys. 49(2):40-47.

15. Gaponov, S. A., and B. N. Smorodsky. 2008. Linear stability of three-dimentional boundary layers. J. Appl. Mech. Techn. Phys. 49(2):157-66.

16. Semionov, N. V., and A.D. Kosinov. 2009. Experiments on stability of supersonic boundary layer on swept wing with "sharp" and "blunted" leading edge. 3rd European Conference for Aerospace Sciences Proceedings. 8 p.

17. Yermolaev, Yu. G., A. D. Kosinov, and N. V. Semionov. 2011. Experimental study of stability of supersonic boundary layer on a swept wing at $\mathrm{M}=2$. TsAGI Sci. J. $\mathrm{XLII}(1): 3-11$.

18. Kosinov, A. D., N. V. Semionov, and Yu. G. Yermolaev. 1999. Disturbances in test section of T-325 supersonic wind tunnel. Novosibirsk: Institute of Theoretical and Applied Mechanics. Preprint No. 6-99. 24 p.

19. Nikias, C.L., and M. R. Raghuveer. 1987. Bispectrum estimation: A digital signal processing framework. Proc. IEEE 75(7):23-45.

20. Kendall, J. M., and R. L. Kimmel. 1991. Nonlinear disturbances in hypersonic laminar boundary layer. AIAA Paper No.91-0320.

21. Kosinov, A.D., and A.I. Semisynov. 2003. Character of evolution of natural disturbances in a supersonic boundary layer on a flat plate. Thermophys. Aeromech. 10(1):39-44. 
22. Chokani, N., D.A. Bountin, A. N. Shiplyuk, and A. A. Maslov. 2005. Nonlinear aspects of hypersonic boundary-layer stability on a porous surface. AIAA J. 43(1):149-55.

23. Lvovskiy, E. N. 1982. Statistical methods for constructing the empirical formulas. Moscow. 224 p. [In Russain.]

24. Kohama, Y., W.S. Saric, and J. A. Hoos. 1991. A high-frequency, secondary instability of crossflow vortices that leads to transition. RAS Conference BoundaryLayer Transition and Control Proceedings. 4.1-4.13.

25. Wassermann, P., and M. Kloker. 2003. Transition mechanisms induced by travelling crossow vortices in a three-dimensional boundary layer. J. Fluid Mech. 483:67-89.

26. White, E., and W. Saric. 2005. Secondary instability of crossow vortices. J. Fluid Mech. 525:275-308. 